\title{
Protective effect of 3-(naphthalen-2-yl(propoxy)methyl)azetidine hydrochloride on hypoxia-induced toxicity by suppressing microglial activation in BV-2 cells
}

\author{
Jiae Kim ${ }^{1, \#}$, Su-Min Kim ${ }^{2, \#}$, Jung-Min $\mathrm{Na}^{1}$, Hoh-Gyu Hahn ${ }^{3}$, Sung-Woo Cho ${ }^{1, *}$ E Seung-Ju Yang ${ }^{2, *}$ \\ ${ }^{1}$ Department of Biochemistry and Molecular Biology, University of Ulsan College of Medicine, Seoul 05505, ${ }^{2}$ Department of Biomedical \\ Laboratory Science, Konyang University, Daejeon 35365, ${ }^{3}$ Chemical Kinomics Research Center, Korea Institute of Science and Technology, \\ Seoul 02456, Korea
}

\begin{abstract}
We recently reported the anti-inflammatory effects of 3-(naphthalen-2-yl(propoxy)methyl)azetidine hydrochloride (KHG26792) on the ATP-induced activation of the NFAT and MAPK pathways through the P2X7 receptor in microglia. To further investigate the underlying mechanism of KHG26792, we studied its protective effects on hypoxia-induced toxicity in microglia. The administration of KHG26792 significantly reduced the hypoxia-induced expression and activity of caspase-3 in BV-2 microglial cells. KHG26792 also reduced hypoxia-induced inducible nitric oxide synthase protein expression, which correlated with reduced nitric oxide accumulation. In addition, KHG26792 attenuated hypoxiainduced protein nitration, reactive oxygen species production, and NADPH oxidase activity. These effects were accompanied by the suppression of hypoxia-induced protein expression of hypoxia-inducible factor 1-alpha and NADPH oxidase-2. Although the clinical relevance of our findings remains to be determined, these data results suggest that KHG26792 prevents hypoxia-induced toxicity by suppressing microglial activation. [BMB Reports 2016; 49(12): 687-692]
\end{abstract}

\section{INTRODUCTION}

The brain is extremely susceptible to hypoxia-induced injury due to its high demand for oxygen; thus, ischemic stroke is

*Corresponding authors. Sung-Woo Cho, Tel: +82-2-3010-4278; Fax: +82-2-3010-4278; E-mail: swcho@amc.seoul.kr, Seung-Ju Yang, Tel: +82-42-600-6372; Fax: +82-42-600-6372; E-mail: sjyang@konyang. ac.kr

${ }^{\#}$ These authors contributed equally to this work.

https://doi.org/10.5483/BMBRep.2016.49.12.169

Received 6 October 2016, Revised 17 October 2016, Accepted 19 October 2016

Keywords: Hypoxia, Microglia, Oxidative stress, 3-(Naphthalen-2yl(propoxy)methyl)azetidine hydrochloride one of the most common brain diseases (1). Microglial activation in the brain plays an important role in the progression of neurodegenerative pathologies; activated microglia secrete reactive oxygen species (ROS), chemokines, and pro-inflammatory cytokines that elicit neuroinflammatory responses and contribute to hypoxic brain injury (2). Because hypoxia can also promote neuronal injury through microglial activation during cerebral ischemia, inflammation may play an important role in the progression of both ischemic stroke and hypoxia (3), although the exact mechanism for microglial activation after ischemic injury remains unclear. Thus, pharmacological agents against microglial activation may have a therapeutic benefit in the amelioration of hypoxic damage. Despite the abundance of data on the subject, neuroprotective drugs that are fully effective against hypoxia-induced microglial activation are yet to be developed.

Azetidine derivatives have been suggested as useful therapeutic agents for various diseases, and a diverse collection of azetidine-based scaffolds for the development of central nervous system (CNS)-focused lead-like libraries have been reported $(4,5)$. In addition, biological activities of azetidine derivatives against serotonin, norepinephrine, and dopamine transporters have been reported $(6,7)$. However, their mechanisms of action are not completely understood.

We have synthesized and examined various compounds for their ability to control neuronal injury related to microglial activation. During the course of these studies, we recently reported the anti-inflammatory effects of 3-(naphthalen-2yl(propoxy)methyl)azetidine hydrochloride (KHG26792) on the ATP-induced activation of the NFAT and MAPK pathways through the P2X7 receptor in microglia (8). However, the mechanism of action of KHG26792 remained largely unclear. Herein we describe the characterization of the effects and mechanisms of KHG26792 on hypoxia-induced toxicity in murine microglia BV-2 cells which reproduce the in vivo situation or primary microglia with high fidelity in many experimental settings, including pharmacological studies (9).

ISSN: 1976-670X (electronic edition)

Copyright (C) 2016 by the The Korean Society for Biochemistry and Molecular Biology

(c) This is an open-access article distributed under the terms of the Creative Commons Attribution Non-Commercial License (http://creativecommons.org/licenses/by-nc/4.0) which permits unrestricted non-commercial use, distribution, and reproduction in any medium, provided the original work is properly cited. 


\section{RESULTS AND DISCUSSION}

\section{KHG26792 alleviates hypoxia-induced toxicity in BV-2 microglial cells}

Exposure to hypoxia induces microglia activation during cerebral ischemia together with production of various pro-inflammatory cytokines and ROS. In this study, we aimed to clarify the protective effect and possible mechanism of KHG26792 on microglial activation induced by hypoxia using in vitro systems.

First, we investigated the effect of KHG26792 on hypoxiainduced toxicity using the MTT assay in cultured BV-2 cells. BV-2 cells were pretreated with KHG26792 for $30 \mathrm{~min}$ followed by hypoxia for $24 \mathrm{~h}$. The results of this assay demonstrated a $49 \%$ reduction in cell viability $24 \mathrm{~h}$ after hypoxia (Fig. 1A), which is in agreement with previous observations (2, 10). However, $50 \mu \mathrm{M}$ KHG26792 rescued

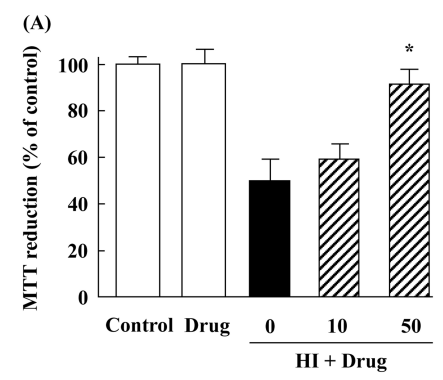

(B)
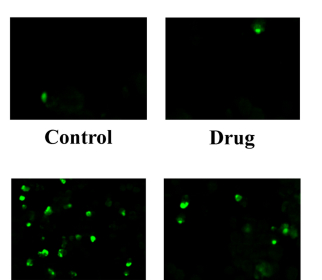

HI

HI +

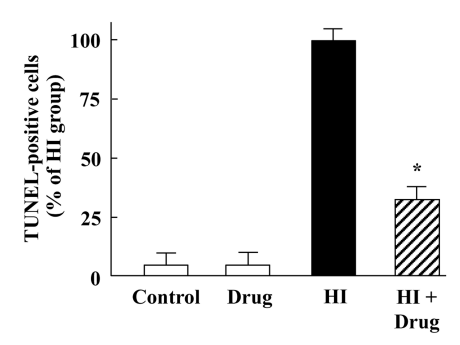

Fig. 1. Effects of KHG26792 on cell viability in hypoxia-induced (HI) BV-2 microglial cells. (A) Cell viability was assessed by the MTT reduction assay. (B) TUNEL assay. Scale bars indicate 10 $\mu \mathrm{m}$. Data are presented as means \pm S.D. and are representative of three independent experiments. *indicates statistical significance between the hypoxia-induced group and hypoxia-induced group pretreated with KHG26792 ( $<<0.01)$. cells from hypoxia-induced toxicity to near normal viability levels (Fig. 1A). No toxicity from KHG26792 itself was observed, and the viability of microglial cells treated with KHG26792 alone approached 100\% (Fig. 1A). The effects of KHG26792 on the hypoxia-induced apoptosis were also confirmed by observing increased TUNEL staining. Our results showed that hypoxia caused widespread TUNEL staining, while no significant TUNEL staining was seen in the control group, suggesting that hypoxia induced apoptotic cell death (Fig. 1B). Interestingly, KHG26792 treatment significantly reduced the hypoxia-induced TUNEL staining (Fig. 1B), suggesting its function as an effective regulator of the apoptotic process and confirming the results obtained by caspase assay.

Previous studies have reported that hypoxia-ischemiainduced apoptosis in the brain is evident by the activation of caspase-3 (10). We further examined the effects of KHG26792 on the levels of caspase-3 as hypoxia is known to promote cell

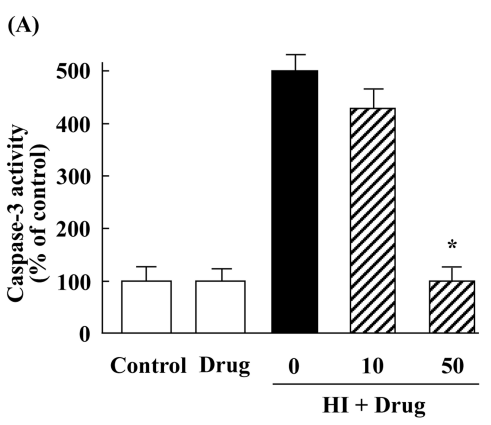

(B)
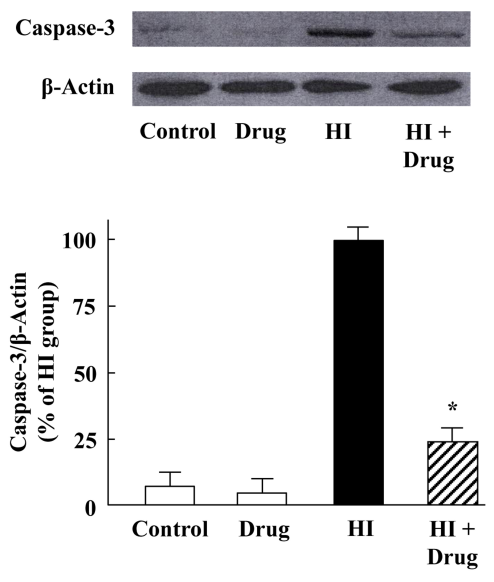

Fig. 2. Effects of KHG26792 on activity and protein level of caspase-3 in hypoxia-induced (HI) BV-2 microglial cells. KHG26792 significantly reduced hypoxia-induced caspase-3 activity (A) and protein levels (B) in BV-2 cells at $24 \mathrm{~h}$ after hypoxia. Data are presented as means \pm S.D. and are representative of three independent experiments. *indicates statistical significance between the hypoxia-induced group and hypoxia-induced group pretreated with KHG26792 ( $\mathrm{P}<0.01)$. 
death through a caspase-3 dependent pathway (11). In this study, KHG26792 pretreatment significantly reduced hypoxiainduced caspase-3 activity (Fig. 2A), further supporting its protective role. Taken together, these results indicate that KHG26792 effectively protected BV-2 cells from hypoxiainduced toxicity. As shown in Fig. 2B, western blot analysis also indicated that hypoxia led to an increased expression of caspase-3, whereas KHG26792 significantly decreased the expression of caspase- 3 in hypoxia-induced BV-2 cells. These results suggest that KHG26792 promotes cell survival via the inhibition of hypoxia-induced expression of caspase-3.

\section{Effects of KHG26792 on hypoxia-induced NO, iNOS, protein} nitration, ROS, and NADPH oxidase

Hypoxia-induced microglial activation releases large quantities of $\mathrm{NO}$ through an increase in iNOS expression in response to brain damage (2). Herein we examined the effects of KHG26792 on the regulation of $\mathrm{NO}$ and iNOS levels in hypoxia-induced BV-2 cells. Hypoxia substantially increased the levels of nitrite, an indicator of $\mathrm{NO}$ production, compared with those in the untreated control (Fig. 3A). Nitrite levels significantly decreased from the pretreatment of KHG26792 compared with those in the corresponding hypoxia-induced group (Fig. 3A). Hypoxia induced a marked upregulation of iNOS protein expression of up to 4 fold (Fig. 3B). In contrast, KHG26792 treatment effectively reduced the levels of iNOS protein compared with those in the hypoxia-induced group in the absence of KHG26792 (Fig. 3B). This reduction in iNOS expression correlated with reduced $\mathrm{NO}$ accumulation. Again, KHG26792 alone showed no significant effects on hypoxiainduced changes in $\mathrm{NO}$ and iNOS levels in BV-2 cells (Fig. 3A, B). Recently, we reported that KHG26792 exhibits antiinflammatory and anti-oxidant activities as a consequence of iNOS downregulation in ATP-induced microglia (8), which is consistent with the anti-inflammatory and anti-oxidant activities of KHG26792 we observed as a consequence of the downregulation of iNOS (Fig. 3B). Collectively, the reduction in the levels of hypoxia-induced $\mathrm{NO}$, along with reduced expression of iNOS, from KHG26792 treatment suggests that KHG26792 is an effective inhibitor of hypoxia-induced injury.

Nitrotyrosine, formed by the reaction of reactive nitrogen species with proteins, is reportedly elevated in hypoxicischemic brain injury (11). To assess the effects of KHG26792 against hypoxia-induced protein nitration, we evaluated nitrotyrosine using immunochemical methods. Hypoxia increased 3-nitrotyrosine levels (Fig. 3C), whereas the treatment of cells with KHG26792 inhibited hypoxia-induced protein nitration (Fig. 3C). KHG26792 itself showed no effects on protein carbonyls or 3-nitrotyrosine levels. These results indicate the potential of KHG26792 in hypoxia-induced protein nitration in cultured BV-2 cells.

ROS induced by hypoxia leads to microglial cell death, and ROS levels have been reported to be increased in the brains of experimental animal models of agent-induced hypoxia (12).
For these reasons, anti-oxidants that suppress hypoxia-induced ROS production may have neuroprotective potential. In the present study, we measured the effects of KHG26792 on the levels of ROS. Consistent with previous reports $(12,13)$, we found that ROS levels in the hypoxia-induced group were approximately 5-fold higher than those in the control group (Fig. 3D). However, KHG26792 administration significantly reduced the high levels of ROS induced by hypoxia (Fig. 3D), suggesting a potential anti-oxidant role of KHG26792.

Previous studies have reported that the activation of NOX serves as a critical source of superoxide, a highly damaging ROS in oxidative injury, and that long-term hypoxia exposure induces excessive brain NOX activity, which plays a role in hypoxia-induced CNS dysfunction and neurobehavioral impairments (14). In addition, NOX-mediated oxidative damage is involved in the pilocarpine model of temporal lobe epilepsy and in the postsynaptic density after transient cerebral ischemia and reperfusion (13). Accordingly, we examined the activity of NOX in hypoxia-induced BV-2 cells. NOX activity was elevated in hypoxia-induced BV-2 cells compared with that in untreated control cells, providing a functional role of
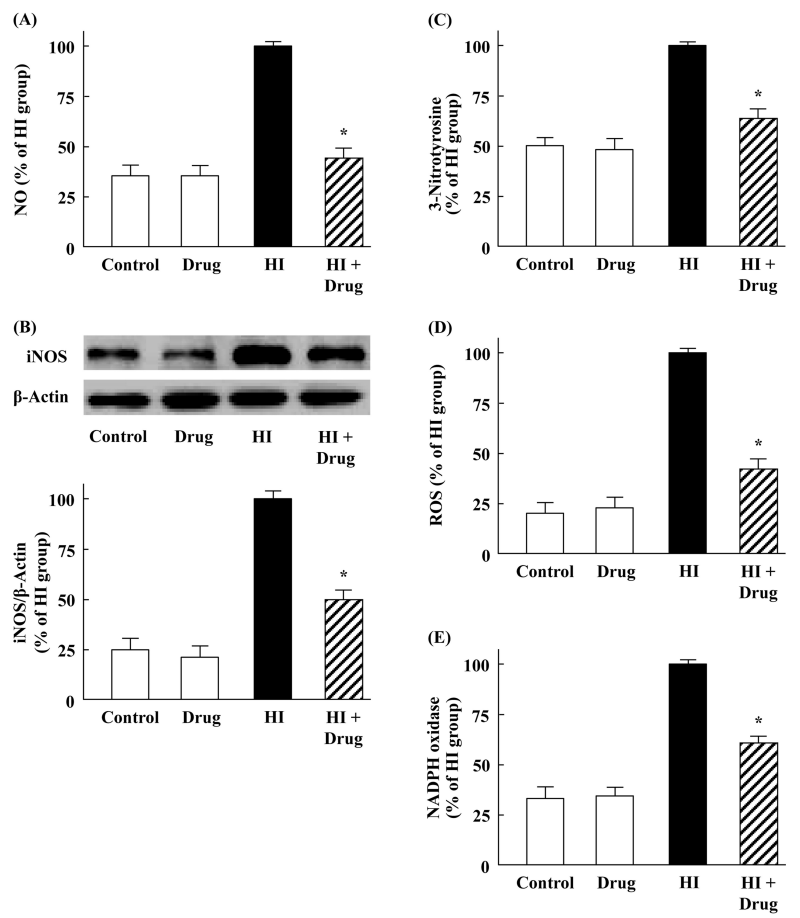

Fig. 3. KHG26792 attenuates hypoxia-induced $(\mathrm{HI})$ increases in NO (A), iNOS protein level (B), protein nitration (C), ROS production (D), and NOX activity (E) in BV-2 cells. Data are presented as means \pm S.D. and are representative of three independent experiments. ${ }^{*}$ indicates statistical significance between the hypoxia-induced group and hypoxia-induced group pretreated with KHG26792 $(\mathrm{P}<0.01)$. 
NOX activation in hypoxia-induced oxidative stress (Fig. 3E). However, the administration of KHG26792 markedly reduced NOX activity (Fig. 3E). These results suggest that KHG26792 reduces the hypoxia-induced accumulation of ROS through an at least partial downregulation of NOX function. Taken together, the results of Fig. 3 demonstrate that KHG26792 may be a valuable and novel agent for studying the relationship between oxidative and disease pathophysiology.

\section{Effects of KHG26792 on hypoxia-induced expression of NOX2, HIF-1 $\alpha$, and NF-kB}

Under hypoxic conditions, increased NOX activity was reportedly accompanied by NOX2 expression in the prefrontal cortex of mice, which also activated HIF-1 in a ROSdependent manner $(12,15)$. In the present study, KHG26792 effectively attenuated hypoxia-induced NOX activity (Fig. 3E) and ROS production (Fig. 3D); thus, we further examined the effect of KHG26792 on the protein expression of NOX2 in hypoxia-induced BV-2 cells. As shown in Fig. 4A, NOX2 was expressed in microglia, consistent with the previous observation by Harrigan et al. (16), but the basal expression of NOX2 was very low in control BV-2 cells. In contrast to the control group, hypoxia dramatically increased NOX2 expression, and the pretreatment of KHG26792 significantly reduced hypoxiainduced NOX2 expression (Fig. 4A, B). These results indicate
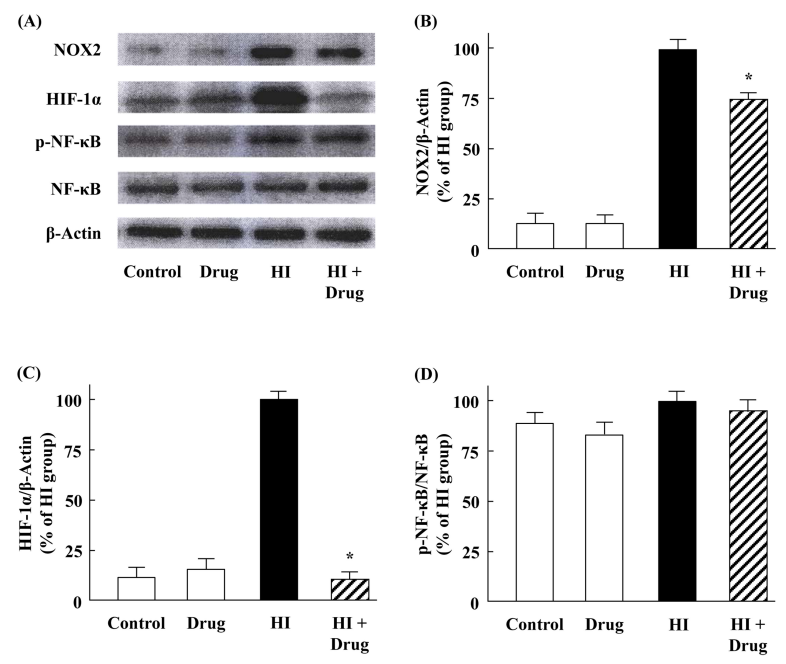

Fig. 4. Effects of KHG26792 on the protein expression levels of NOX2, HIF- $1 \alpha$, and NF- $\mathrm{KB}$ in hypoxia-induced (HI) BV-2 microglial cells. Equal amounts of crude extracts were immunoblotted using primary antibodies against each protein (A). Bar graphs showing the quantification of levels of NOX2/ $\beta$-actin, HIF- $1 \alpha / \beta$-actin, and phospho-NF- $\kappa \mathrm{B} / \mathrm{NF}-\kappa \mathrm{B}$ were calculated using densitometry (B-D). Data are presented as means \pm S.D. and are representative of three independent experiments. *indicates statistical significance between the hypoxia-induced group and hypoxia-induced group pretreated with KHG26792 (P $<0.01)$. that NOX2-derived oxidative stress is involved in hypoxiainduced microglial activation, and the suppression of NOX2 expression by KHG26792 may partly explain its anti-oxidant and anti-apoptotic properties in BV-2 cells under hypoxic conditions.

HIF-1 regulates oxygen homeostasis and is composed of two transcription factors: HIF- $1 \alpha$ and HIF-1 $\beta$. Unlike HIF- $1 \beta$, which is constitutively present, HIF- $1 \alpha$ is kept at low levels in many cell types under normoxia. Many studies have shown that hypoxia may lead to the activation of microglia and the induced expression of HIF- $1 \alpha$ was readily detected in hypoxic BV-2 cells (17). Previous studies have also demonstrated that HIF-1 $\alpha$ has a pro-oxidant role under hypoxic conditions by increasing NOX activity (15). Moreover, HIF- $1 \alpha$ directly binds to the promoter of NOX2 and is required for hypoxia-induced ROS generation (12). In the present study, we investigated the effect of KHG26792 on the induced expression of HIF-1 $\alpha$ in BV-2 cells.

As shown in Fig. 4, hypoxia increased the protein expression of HIF- $1 \alpha$, which is consistent with previous reports (17). Increased HIF- $1 \alpha$ expression was effectively attenuated by pretreatment with KHG26792. Taken together, the downregulation of NOX2 and HIF-1 $\alpha$ (Fig. 4) was accompanied by a decrease in ROS levels (Fig. 3). These results suggest that the suppression of NOX2/HIF- $1 \alpha$ signaling is involved in the protective effect of KHG26792 against hypoxia-induced ROS production in microglial cells, although the precise mechanism of ROS generation under hypoxic conditions remains unclear. Our results provide further insight into the molecular mechanisms underlying the regulation of the oxygen sensitivity of HIF and into the development of agents that manipulate HIF activity as therapeutic targets for hypoxiainduced disorders in CNS (18).

Many studies have reported that NF- $\kappa \mathrm{B}$ can be activated by hypoxia. Thus, we next explored whether KHG26792 could prevent the activation of the NF-KB pathway in hypoxiainduced BV-2 cells. Western blotting results indicate that the levels of NF-kB expression were not significantly changed in any group but that hypoxia slightly increased the phosphorylation of NF- $\mathrm{KB}$ (Fig. 4A, B), which is in agreement with previous observations (2). However, no significant changes in the phosphorylation of NF-kB were observed among the hypoxia-induced groups in the presence or absence of KHG26792 (Fig. 4A, B), suggesting that KHG26792 did not inhibit NF- $\mathrm{B} B$ activation.

In summary, our present report is the first to show that KHG26792 can significantly attenuate hypoxia-induced oxidative stress in microglia. However, we cannot exclude other potential mechanisms by which KHG26792 may attenuate hypoxia-induced microglial cell injury. Our findings in this study add to the anti-oxidative potential of KHG26792 for use in brain injury, although further supporting data from in vivo studies may need to clarify the mechanisms involved and to further understand the protective effects of this novel 
compound.

\section{MATERIALS AND METHODS}

\section{Materials}

Phosphate-buffered saline and dimethyl sulfoxide (DMSO) were obtained from Sigma-Aldrich (St. Louis, MO). Anti-inducible nitric oxide synthase (iNOS), anti-caspase-3, anti-NF-kB p65, anti-phospho-NF-kB p65, anti-hypoxia-inducible factor (HIF)-1 alpha, anti-NADPH oxidase 2 (NOX2), and anti- $\beta$-actin were purchased from Cell Signaling Technology (Beverly, MA). All other reagents were of the highest purity among commercially available products. KHG26792 was synthesized and purified as described previously (19). KHG26792 was dissolved in DMSO and stored at $-20^{\circ} \mathrm{C}$ as a stock solution $(10 \mathrm{mM})$. KHG26792 was then diluted to the desired final concentration in the treatment medium.

\section{Cell culture and hypoxic exposure}

BV-2 microglial cells were maintained in DMEM with 5\% FBS, $2 \mathrm{mM}$ L-glutamine, $100 \mathrm{U} / \mathrm{ml}$ penicillin, and $100 \mathrm{mg} / \mathrm{ml}$ streptomycin (Sigma-Aldrich, St. Louis, $\mathrm{MO}$ ) in a $5 \% \mathrm{CO}_{2}$ incubator, as described previously (8). For hypoxic exposure, cells were changed to hypoxia by placing them in a chamber with $3 \%$ oxygen, $5 \% \mathrm{CO}_{2}$, and $92 \%$ nitrogen at $37^{\circ} \mathrm{C}$. Cells serving as controls were incubated at $37^{\circ} \mathrm{C}$ with $95 \%$ air and $5 \%$ CO2 (2). For most experiments, BV-2 cells were pretreated with KHG26792 for 30 min followed by hypoxia for $24 \mathrm{~h}$, while controls were treated with the vehicle except where indicated. Cell viability was determined by 3-(4,5-dimethylthiazol-2-yl)-2,5-diphenyltetrazoliumbromide sodium (MTT) assay, as described previously (20). The dark blue formazan crystals that formed in intact cells were solubilized with MTT lysis buffer, and absorbance at $595 \mathrm{~nm}$ was measured using an enzyme-linked immunosorbent assay (ELISA) microplate reader.

\section{TUNEL staining}

Terminal deoxynucleotidyl transferase-mediated dUTP nickend labeling (TUNEL) assay was performed in accordance with manufacturer specifications with a slight modification as described elsewhere (21). The DeadEnd Fluorometric TUNEL system (Promega, Madison, WI, USA) was used for nick-end labeling with terminal deoxynucleotidyl transferase. The TUNEL positive cells with green fluorescent staining were defined as apoptotic cell death. For cell counts, TUNELpositive cells were counted manually in three different images of each of three coverslips by three individuals blinded to the experiment.

\footnotetext{
Measurement of caspase- 3 and NADPH oxidase activity

For caspase-3 assay, cell lysates were incubated with the caspase-3 substrate acetyl-DEVD-p-nitroanilide (Ac-DEVDpNA; Sigma) at $37^{\circ} \mathrm{C}$ for $1 \mathrm{~h}$ in $30 \mathrm{mM}$ HEPES, $100 \mathrm{mM} \mathrm{NaCl}$, $0.15 \%$ Triton $\mathrm{X}-100,1 \mathrm{mM}$ protease inhibitor cocktail (Sigma),
}

$0.3 \mathrm{mM}$ EDTA, and $10 \mathrm{mM}$ DTT. The changes in optical density at $405 \mathrm{~nm}$ were measured using a spectrophotometer. NADPH oxidase (NOX) activity was estimated depending on the oxidation of dihydroethidium to ethidium, as described previously (2). Ethidium fluorescence was measured over 30 min at an excitation wavelength of $480 \mathrm{~nm}$ and an emission wavelength of $610 \mathrm{~nm}$. NOX activity was expressed as the change in ethidium fluorescence per hour and was then normalized to the value for the hypoxia-induced group.

\section{Protein nitration}

3-Nitrotyrosine levels were immunochemically measured using the methods described by Sultana et al. (22). The mouse anti-nitrotyrosine antibody was used as the primary antibody, and immunoreactive bands were detected using an enhanced chemiluminescence kit according to the manufacturer's instructions (Amersham Bioscience, Piscataway, NJ). Densitometric analyses of bands in blot images were used to calculate the levels of 3-nitrotyrosine.

\section{Measurement of nitric oxide (NO) and reactive oxygen species (ROS) Production}

Because nitrite is a major product of $\mathrm{NO}$, the concentration of $\mathrm{NO}$ in supernatants was assessed by measuring the amount of nitrite generated using Griess reagent [ $1 \%$ sulfanilamide/ $0.1 \%$ $\mathrm{N}$-(1-naphthyl)-ethylenediamine dihydrochloride/2.5\% $\left.\mathrm{H}_{3} \mathrm{PO}_{4}\right]$, as previously described (8). Optical density was measured at $540 \mathrm{~nm}$ using a microplate reader. The NO concentration was calculated based on a reference standard curve for sodium nitrite generated using known concentrations of NO.

A microfluorescence assay using DCF-DA was used to monitor the production of ROS in BV-2 cells, as described previously (8). The fluorescence intensity of the DCF product was measured by using a SpectraMax GEMINI XS fluorescence spectrophotometer (Molecular Devices) at an excitation wavelength of $485 \mathrm{~nm}$ and an emission wavelength of 538 $\mathrm{nm}$. All experiments were performed in the dark.

\section{Western blotting}

Crude extracts were mixed with sodium dodecyl sulfatepolyacrylamide gel electrophoresis (SDS-PAGE) loading buffer, boiled for $5 \mathrm{~min}$, and analyzed by $10 \%$ SDS-PAGE. Resolved proteins were transferred onto nitrocellulose membranes, detected by enhanced chemiluminescence according to the manufacturer's instructions (Amersham, Buckinghamshire, UK), and analyzed using a Molecular Image ChemiDoc XRS system (Bio-Rad; Hercules, CA) (23). Densitometry was performed using Image J software (NIH; Bethesda, MD). $\beta$-Actin was used to confirm equal protein loading for all samples.

\section{Statistical analysis}

Statistical data are expressed as means \pm SD and are representative of three independent experiments. Individual differences between the groups were analyzed using one-way 
ANOVA, followed by Student's t-test, and a threshold of P $<$ 0.01 was used to define statistical significance.

\section{ACKNOWLEDGEMENTS}

This study was supported by the Basic Science Research Program through the National Research Foundation of Korea (NRF) funded by the Ministry of Education (2015R1D1A1A 09056947 and 2015R1D1A3A01015793) and by Student Research Grants from the University of Ulsan College of Medicine (2015-2016), Seoul, Korea.

\section{REFERENCES}

1. Shi H, Sheng B, Zhang F et al (2013) Kruppel-like factor 2 protects against ischemic stroke by regulating endothelial blood brain barrier function. Am J Physiol Heart Circ Physiol 304, H796-H805

2. Zhang Q, Yuana L, Zhang Q et al (2015) Resveratrol attenuates hypoxia-induced neurotoxicity through inhibiting microglial activation. Int Immunopharmacol 28, 578-587

3. Chamorro A and Hallenbeck J (2006) The harms and benefits of inflammatory and immune responses in vascular disease. Stroke 37, 291-293

4. Lowe JT, Lee MD, Akella LB et al (2012) Synthesis and profiling of a diverse collection of azetidine-based scaffolds for the development of CNS-focused lead-like libraries. J Org Chem 77, 7187-7211

5. Mnich SJ, Hiebsch RR, Huff RM and Muthian S (2010) Anti-inflammatory properties of CB1-receptor antagonist involves beta2 adrenoceptors. J Pharmacol Exp Ther 333, 445-453

6. Han M, Song C, Jeong N and Hahn HG (2014) Exploration of 3-aminoazetidines as triple reuptake inhibitors by bioisosteric modification of 3-aoxyazetidine. ACS Med Chem Lett 5, 999-1004

7. Yun J, Han M, Song C, Cheon SH, Choi K and Hahn HG (2014) Synthesis and biological evaluation of 3phenethylazetidine derivatives as triple reuptake inhibitors. Bioorg Med Chem Lett 24, 3234-3237

8. Kim EA, Cho CH, Kim J et al (2015) The azetidine derivative, KHG26792 protects against ATP-induced activation of NFAT and MAPK pathways through P2X7 receptor in microglia. Neurotoxicology 51, 198-206

9. Henn A, Lund S, Hedtjärn M, Schrattenholz A, Pörzgen P and Leist M (2009) The suitability of BV2 cells as alternative model system for primary microglia cultures or for animal experiments examining brain inflammation. Altex 26, 83-94

10. Hou CW, Chen YL, Chuang SH, Wang JS and Jeng KC (2014) Protective effect of a sesamin derivative, 3-bis (3-methoxybenzyl) butane-1, 4-diol on ischemic and hypoxic neuronal injury. J Biomed Sci 21, 15

11. Doverhag C, Hedtjärn M, Poirier F et al (2010) Galectin-3 contributes to neonatal hypoxic-ischemic brain injury. Neurobiol Dis 38, 36-46

12. Guan D, Su Y, Li Y et al (2015) Tetramethylpyrazine inhibits $\mathrm{CoCl} 2$-induced neurotoxicity through enhancement of $\mathrm{Nrf} 2 / \mathrm{GCL} / \mathrm{GSH}$ and suppression of HIF1 $\alpha / \mathrm{NOX} 2 / \mathrm{ROS}$ pathways. J Neurochem 134, 551-565

13. Li H, Wang Y, Feng D et al (2014) Alterations in the time course of expression of the Nox family in the brain in a rat experimental cerebral ischemia and reperfusion model: effects of melatonin. J Pineal Res 57, 110-119

14. Nair D, Dayyat EA, Zhang SX, Wang Y and Gozal D (2011) Intermittent hypoxia-induced cognitive deficits are mediated by NADPH oxidase activity in a murine model of sleep apnea. PLoS One 6, e19847

15. Yuan G, Khan SA, Luo W, Nanduri J, Semenza GL and Prabhakar NR (2011) Hypoxia-inducible factor 1 mediates increased expression of NADPH oxidase-2 in response to intermittent hypoxia. J Cell Physiol 226, 2925-2933

16. Harrigan TJ, Abdullaev IF, Jourd'heuil D and Mongin AA (2008) Activation of microglia with zymosan promotes excitatory amino acid release via volume-regulated anion channels: the role of NADPH oxidases. J Neurochem 106, 2449-2462

17. Yao L, Kan EM, Lu J et al (2013) Toll-like receptor 4 mediates microglial activation and production of inflammatory mediators in neonatal rat brain following hypoxia: role of TLR4 in hypoxic microglia. J Neuroinflammation 10, 23

18. Freeman RS and Barone MC (2005) Targeting hypoxiainducible factor (HIF) as a therapeutic strategy for CNS disorders. Curr Drug Targets CNS Neurol Disord 4, 85-92

19. Han Y, Han M, Shin D, Song C and Hahn HG (2012) Exploration of novel 3-substituted azetidine derivatives as triple reuptake inhibitors. J Med Chem 55, 8188-8192

20. Lee D, Kook S-H, Ji H et al (2015) N-acetyl cysteine inhibits $\mathrm{H} 2 \mathrm{O} 2$-mediated reduction in the mineralization of MC3T3-E1 cells by down-regulating Nrf2/HO-1 pathway. BMB Rep 48, 636-641

21. Choi SH, Park BK, Lee KW, Chang J, Lee $Y$ and Kwon HJ (2015) Effect of respiratory syncytial virus on the growth of hepatocellular carcinoma cell-lines. BMB Rep 48, 565-570

22. Sultana R, Ravagna A, Mohmmad-Abdul H, Calabrese $V$ and Butterfield DA (2005) Ferulic acid ethyl ester protects neurons against amyloid beta-peptide(1-42)-induced oxidative stress and neurotoxicity: relationship to antioxidant activity. J Neurochem 92, 749-758

23. Eom SA, Kim DW, Shin MJ et al (2015) Protective effects of PEP-1-Catalase on stress-induced cellular toxicity and MPTP-induced Parkinson's disease. BMB Rep 48, 395-400 